\title{
Spatial patterns in non-locally interacting particle systems
}

\author{
Cristóbal López ${ }^{a}$ and Emilio Hernández-García \\ Instituto de Física Interdisciplinar y Sistemas Complejos (IFISC), CSIC-UIB, Campus de la Universidad \\ de las Islas Baleares, E-07122 Palma de Mallorca, Spain.
}

\begin{abstract}
The influence of spatially non-local interactions on the aggregation, competition, and growth dynamics of interacting particle systems has been recently addressed. In this paper we survey recent results obtained for this kind of systems, focusing on two types of population dynamics models: a) densitydependent mobility particle systems, with conserved total number of individuals, and b) birth-death systems, where annihilation-creation events are allowed, so that the total number of particles is not conserved. Both models present a pattern forming instability leading to surprisingly similar spatial structures. The two levels of description, microscopic-particle and macroscopic-density, are analyzed. From the last one, a clear identification of the pattern forming instability is obtained.
\end{abstract}

\section{Introduction}

Spatial non-local interactions have been usually considered in the study of biological systems since they account for the mutual influence of organisms that are separated in space [1]. With special emphasis, non-locality has been addressed as an important mechanism in the formation of organism aggregates, including fish schools, insect swarms, bird flocks, bacterial patterns and patchy plankton structures. In all these cases, influence-at-a-distance arises from different biological behaviors: detection of preys, visual or hearing stimuli, chemical signaling, and many others that, at the end, characterize the individuals in a given species by a finite range of interaction, $R$. The presence of a characteristic length leads naturally to the possibility of pattern forming phenomena.

This paper surveys recent studies which describe interplay between non-locality and pattern formation. They are in the line of traditional investigations whose focus is to highlight the causes of aggregation, focussing in cluster formation, but here the additional phenomenon of cluster rearrangement leading to periodic patterns is observed. The methodology in these studies is the use of very simple models of discrete particle dynamics that, however, retain some basic mechanisms leading to clustering and ordering. The main assumptions for these models are [2]:

- Particles or agents move in space performing some kind of random walk.

- They interact with other particles through attractive or repulsive forces, or by modifying their velocity because of communication with neighbors, or rather by altering their growth rates in response to the presence of other organisms.

Non-locality may enter in any of the above items. At the level of the random walk, some models have recently considered that the mobility of the particles, i.e. its diffusion coefficient, depends on the number density of other particles within the interaction range [3-5]. On the

\footnotetext{
a e-mail: clopez@imedea.uib.es
} 
other hand, the interplay between non-locality and the interaction among particles (the second item above) is even more obvious: the interaction range is the typical length scale for any of the inter-particle influences mentioned there.

In this work we collect previous results that were presented separately, allowing for a clearer comparison and recognition of similarities and differences. Firstly, we analyze a system of particles where no direct attractive nor repulsive forces among them are present. Moreover, there is no birth-death dynamics and the total number of particles is conserved. Non-locality enters here by assuming that the mobility of a given particle depends on the number of particles surrounding it within a finite distance. This can be interpreted as an effective change of the mobility because of the presence (via demographic pressure, collisions, excluded volume effects, etc...) of other particles. A mean-field density description of the model turns out to be related to models used to describe some instances of animal dispersion processes, as for example insect dispersion [6], but with a distinct non-local form of the density-dependent diffusivity. The aggregation patterns will be analyzed at two levels: particle and density equations.

In the second part, we consider a different particle dynamics, of birth-death type, that does not conserve the total number of individuals. In this case, the non-locality enters in the modification of the rates responding to the population present in a given neighborhood of each particle. Clustering and ordering of particles also occur and will be analyzed at the two levels of description. Comparisons with the former case will be emphasized.

The Paper is organized as follows. Section 2 is devoted to the non-local density-dependent mobility model, which we study in its particle representation (Subsect. 2.1) and in the corresponding mean-field density description (Subsect. 2.2). The non-local birth-death rates model is considered in Sect. 3, also in its particle (Subsect. 3.1) and mean-field density description (Subsect. 3.2). Finally, in Sect. 4 we present our discussion and conclusions.

\section{Non-local density-dependent mobility}

In this section we discuss a model recently introduced in [5] where the particles perform random walk steps in such a way that, at every time step, each particle modifies its step size, and thus its diffusion coefficient, depending on the number of other particles within a distance $R$. In principle we can consider both the cases in which diffusion is enhanced in the regions of dense populations, or the opposite case in which diffusion is slowed there. Here we restrict to the first situation, which is appropriate in some processes of animal dispersion.

\subsection{Discrete particle model}

The system consists of $N$ particles with positions $\mathbf{r}(t)=\left(x_{i}(t), y_{i}(t)\right), i=1,2, \ldots N$, in a twodimensional system of size $L \times L$ (we take $L=1$ ) with periodic boundary conditions. At every time $t$ the positions of all the particles are updated synchronously as follows:

$$
\begin{aligned}
& x_{i}(t+\Delta t)=x_{i}(t)+\sqrt{2 D_{0} \Delta t\left(N_{R}(i) / N\right)^{p}} \eta_{i}^{x}(t), \\
& y_{i}(t+\Delta t)=y_{i}(t)+\sqrt{2 D_{0} \Delta t\left(N_{R}(i) / N\right)^{p}} \eta_{i}^{y}(t),
\end{aligned}
$$

where $D_{0}$ is a constant giving the scale of diffusivity, $\Delta t$ is the time step which we take equal to 1 in the numerical simulations, $\boldsymbol{\eta}=\left(\eta^{x}, \eta^{y}\right)$ is a Gaussian white noise with correlations $\left\langle\eta_{i}^{a}(t), \eta_{j}^{b}\left(t^{\prime}\right)\right\rangle=\delta_{a b} \delta_{i j} \delta_{t t^{\prime}} . N_{R}(i)$ is the number of particles at a distance less than $R$ of particle $i$ at time $t$, and $p$ is a positive real number that determines the dependence of the effective diffusivity, $D_{i}=D_{0}\left(N_{R}(i) / N\right)^{p}$ of particle $i$ on $N_{R}(i)$. Note that the functional dependence of $D_{i}$ is of the type considered in standard models of density-dependent dispersal of insects [6]. In accordance with these cases, in which population pressure increases the mobility of the insects in dense zones, we take always $p$ positive. We note however that the case of negative $p$ was considered in [4] and the clustering dynamics there turned out to be rather different to the one of the present model. The behavior of $D_{i}$ is easily understood in the limits $R \rightarrow 0$ and $R \rightarrow L$. 

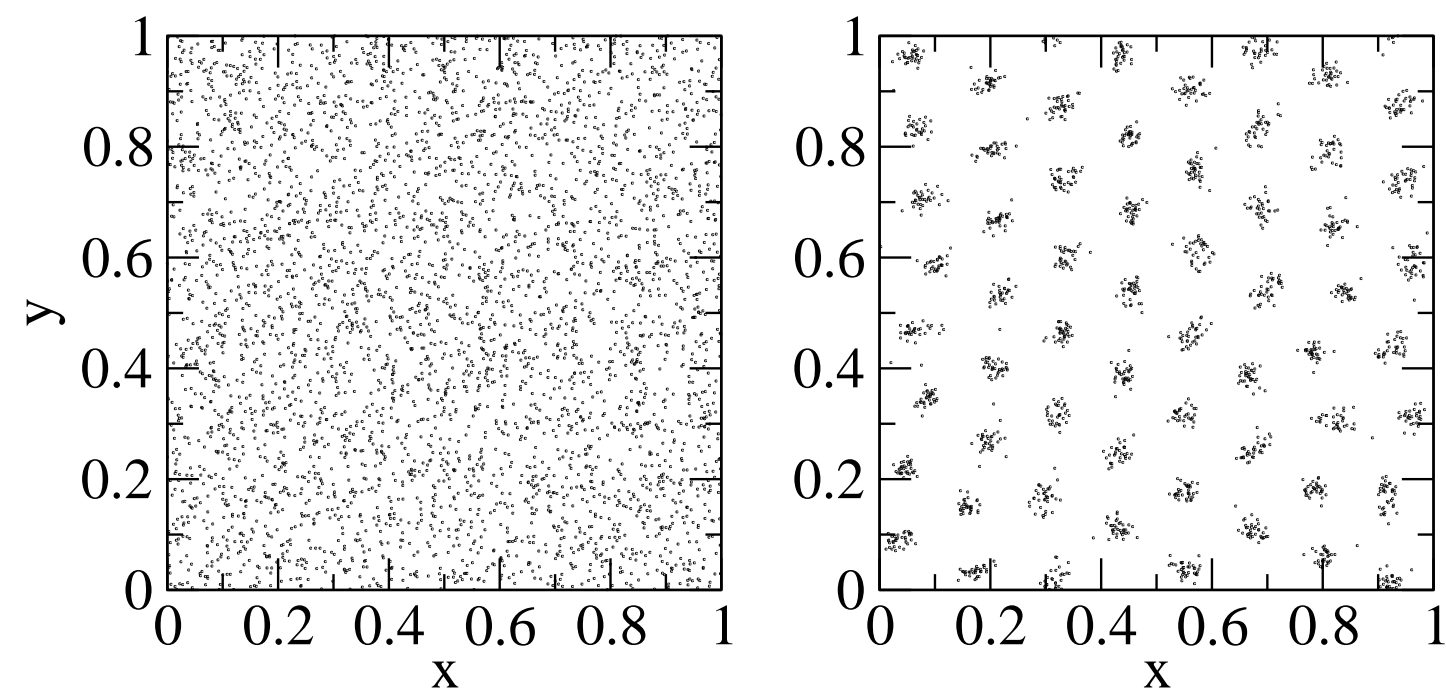

Fig. 1. Instantaneous spatial distributions of particles obtained under the dynamics (1) at long times. Left is for $p=2$, and right for $p=9$. The rest of parameters are, in both plots, $D_{0}=0.0001, R=0.1$, and $N=2000$.

In the first case, $N_{R}(i)=1$ for all $i$ so that all the particles diffuse with the same diffusion coefficient $D_{i}=D_{0}(1 / N)^{p}$, which is negligible for large $N$ so that dynamics freezes in. In the second limit $N_{R}(i)=N$, and now $D_{i}=D_{0}$ for all the particles. In any case, since all particles have the same value of $D_{i}$, no aggregation behavior is expected in these two limits.

Moving $R$ away from these limit values, numerical simulations show that, independently of the value of $D_{0}$, and for large enough $p$, particles aggregate in clusters and the clusters order spatially to form a periodic pattern of hexagonal type and rather well defined wavenumber. In Fig.1 we show the spatial distributions of particles at large time for $p=2$, for which particles are homogeneously distributed in space (left), and $p=9$, for which a pattern of hexagonal symmetry is evident (right). We note that both configurations are highly dynamic, with particles continuously moving, but around mean locations that are well defined and periodically arranged in the second case.

To make a more detailed analysis of the structures attained as a function of the behavioral parameter $p$, which a priori seems to determine the pattern formation transition, we calculated the structure function of the particle spatial distribution at long times for different values of $p$. The structure function is defined as

$$
S_{d}(k)=\left\langle\frac{1}{N}\left|\sum_{j} e^{i \mathbf{k} \cdot \mathbf{r}_{j}(t)}\right|^{2}\right\rangle,
$$

where $\mathbf{r}_{j}(t)$ is the position vector of particle $j, \mathbf{k}$ is a two-dimensional wavevector with modulus $k$, and the average indicates a spherical average over the wavevectors with modulus $k$, a temporal average in the steady state, and an average over many different realizations. In addition to a peak at $k=0$, of height $N$, which reflects the number of particles in the system, the structure function shows an additional peak at a non-vanishing value of $k, k_{M}$, which scales with the interaction range $R$ as $k_{M} \sim 1 / R$, and with a height strongly dependent on the value of $p$. Since maxima of $S_{d}$ identify periodicities in the distributions, the strong change in height of $S_{d}\left(k_{M}\right)$ reflects the transition from homogeneity to pattern formation at that wavenumber. Fig. 2a shows this value $S_{d}\left(k_{M}\right)$ versus the parameter $p$. $S_{d}\left(k_{M}\right)$ remains small for small $p$, indicating an essentially random distribution of particles for any $D_{0}$. For $p \geq p_{c} \approx 7.6$ particles self-organize in a periodic array of clusters. 

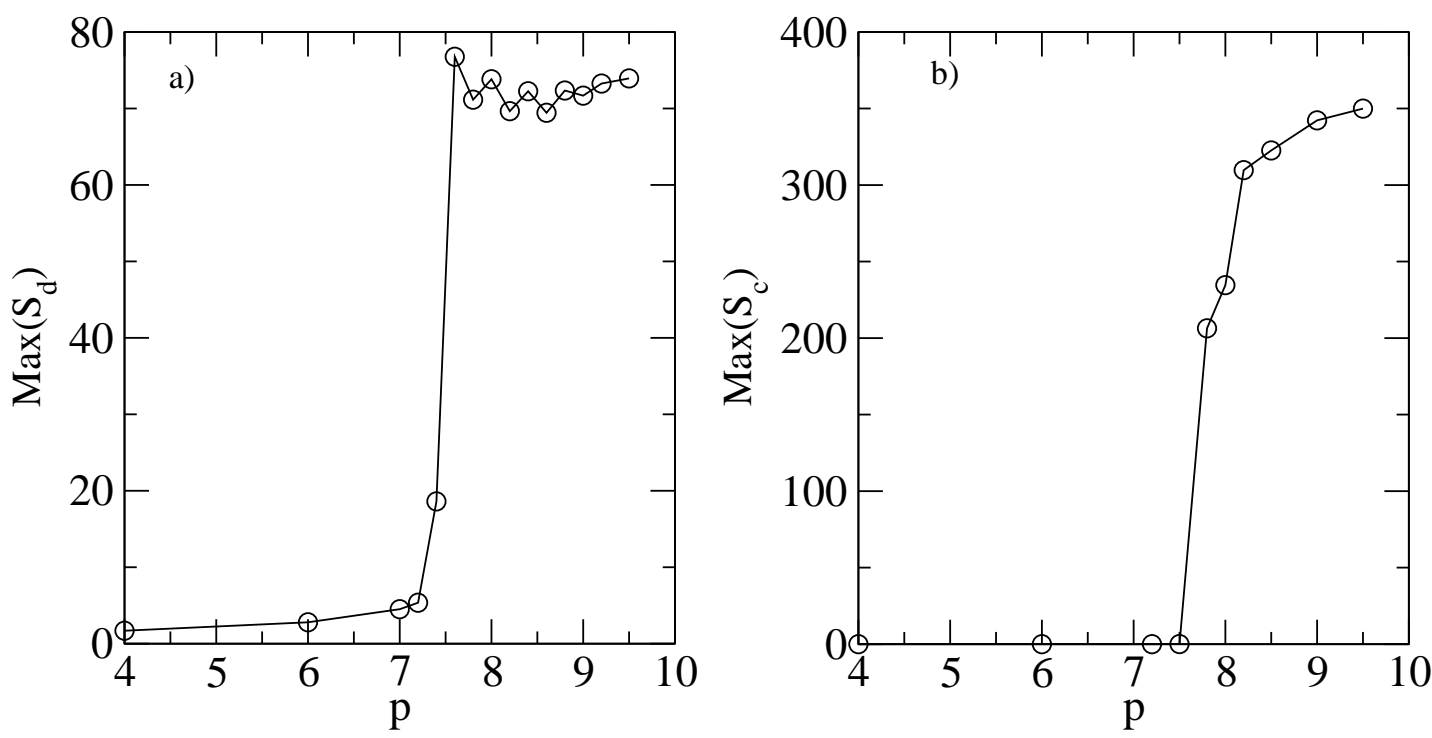

Fig. 2. Characterization of the pattern formation in the nonlocal density-dependent mobility models for $D_{0}=0.0001$ and $R=0.1$. a) Maxima of the particle distribution structure function $\max \left(S_{d}\right)=S_{d}\left(k_{M}\right)$ at long times for different values of the parameter $p . \mathrm{b}$ ) Maxima of the structure function associated to the density field in the continuous model, $\max \left(S_{c}\right)=S_{c}\left(k_{M}\right)$, also as a function of $p$.

The fact that a mechanism of increasing mobility of the particles when their surroundings are crowded leads to clustering, and that the clusters arrange periodically, needs of a clearer explanation. With this aim we next study the density equation for the model.

\subsection{Continuum approach}

To obtain a continuum description of the above process we first take the limit $\Delta t \rightarrow 0$ in Eq.(1) so that we obtain the Langevin equations:

$$
d \mathbf{r}_{i}(t)=\alpha\left(\mathbf{r}_{i}(t), t\right) d \mathbf{W}_{i}(t), \quad i=1, \ldots, N,
$$

where $\alpha\left(\mathbf{r}_{i}(t), t\right)=\sqrt{2 D_{0}\left(N_{R}(i) / N\right)^{p}}$ and $\mathbf{W}_{i}(t)$ are independent and normalized Wiener processes. It is important to note that Eq. (3) should be interpreted in the Ito calculus, since it has been obtained in the continuous-time limit from a non-anticipating discrete-time dynamics.

To derive an equation for the density of particles we use a standard mean-field approach. First we realize that, for each particle $i, \alpha\left(\mathbf{r}_{i}(t), t\right)$ can be though as the value of a space and time dependent noise intensity $\alpha(\mathbf{x}, t)$ evaluated at the position of the particle, $\mathbf{x}=\mathbf{r}_{i}(t)$. Thus, the probability density for the position of particle $i$ (in the Ito interpretation) is $[7,8]$ :

$$
\frac{\partial \rho(\mathbf{x}, t)}{\partial t}=\frac{1}{2} \nabla^{2}\left(\alpha(\mathbf{x}, t)^{2} \rho(\mathbf{x}, t)\right) .
$$

The probability density $\rho(\mathbf{x}, t)$ is also the expected density for an ensemble of independent particles identical to particle $i$. We now close the many body problem at the mean field level by stating that the spatio-temporal dependence of $\alpha(\mathbf{x}, t)$ is self-consistently determined from $\rho: N_{R}(i)=N_{R}(\mathbf{x})=\int_{|\mathbf{x}-\mathbf{r}|<R} d \mathbf{r} \rho(\mathbf{r}, t)$. Thus, the final evolution equation for the expected particle density in our model is

$$
\frac{\partial \rho(\mathbf{x}, t)}{\partial t}=D_{0} \nabla^{2}\left(\rho(\mathbf{x}, t)\left(\frac{1}{N} \int_{|\mathbf{x}-\mathbf{r}| \leq R} d \mathbf{r} \rho(\mathbf{r}, t)\right)^{p}\right),
$$



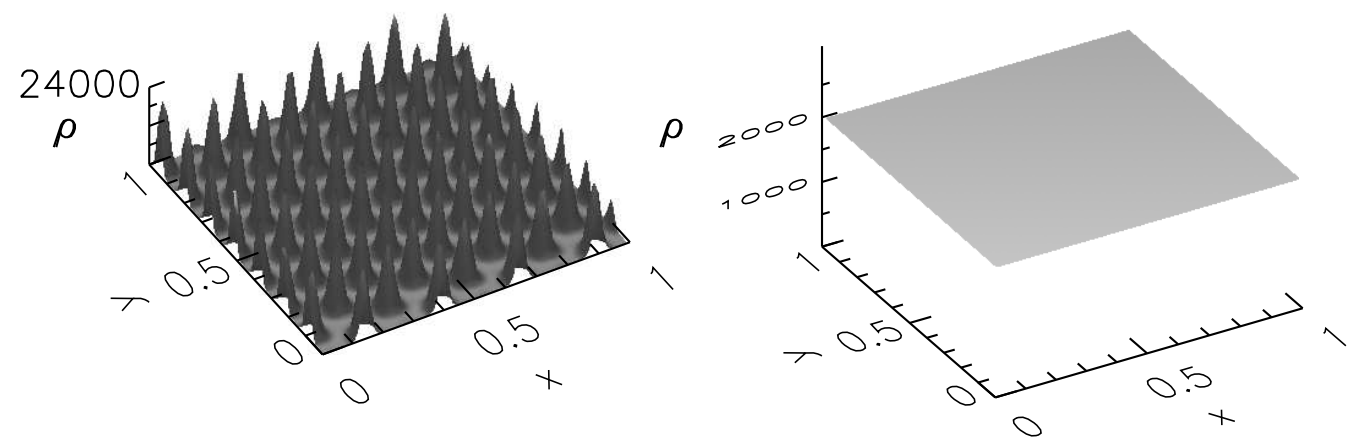

Fig. 3. Steady number density of particles with non-local density-dependence diffusivity from Eq. (5). Left is for $p=9$ and right for $p=2$. The values of the parameters, in both plots, are $R=0.1$, $D_{0}=0.0001, \rho_{0}=N / L^{2}=2000$, and system sizes in the $x$ and $y$ directions are equal to 1 .

A more rigorous derivation of Eq. (5) from the interacting particle dynamics (3), performed for example along the lines of [9] or [10] (see also [11]), gives rise to an additional multiplicative noise term that has been effectively averaged out in Eq. (5). Thus the mean field equation (5) is expected to be a good approximation for not too small densities. In any case, fluctuations in the density equation, which reflect the discrete nature of the particles, seem to have an irrelevant role in the pattern formation instability to be discussed below. It is also important to note that Eq.(5) is a kind of nonlinear diffusion equation with density-dependent diffusion coefficient given by $D(\hat{\rho})=D_{0}\left(\bar{\rho} / \rho_{0}\right)^{p}$ (which is a functional form used in studies of animal dispersal [6]) but with $\bar{\rho}(\mathbf{x}, t)=L^{-2} \int_{|\mathbf{x}-\mathbf{r}| \leq R} d \mathbf{r} \rho(\mathbf{r}, t)$ a kind of averaged density of particles around position $\mathbf{x}$ at time $t$ and $\rho_{0}=N / L^{2}$ is the mean density.

Fig. 3 shows a long-time density solution of Eq.(5) for $p=2$ (right) and $p=9$ (left). The parameters are the same as in Fig.1, and the spatial structures turn out to be the same as in the discrete model, i.e., a hexagonal pattern for large $p$ and a homogeneous density for small $p$. The structure function for the density field is defined by

$$
S_{c}(k)=\left\langle\frac{1}{L^{2}}\left|\int d \mathbf{x} e^{i \mathbf{k} \cdot \mathbf{x}} \rho(\mathbf{x}, t)\right|\right\rangle,
$$

where the average is, as before, a spherical average over the wavevectors with modulus $k$, a temporal average in the steady state, and an average over many different realizations. This definition of structure function is different and can not be directly compared with the discrete expression, Eq. (2), but as in the particle case, $S_{c}$ displays a maximum at a non-vanishing wavenumber $k_{M}$ for $p \geq 7.6$, indicating the appearance of a periodic structure at the same parameter value as in the particle model. This implies that Eq. (5) gives a very good approximation to the particle dynamics. The maximum of $S_{c}$ vs $p$ is plotted in fig. $2 \mathrm{~b}$.

We can, therefore, make a further analytic study of the model. We perform a linear stability analysis of the stationary homogenous solutions of Eq.(5), $\rho_{0}$, by adding a small perturbation: $\rho(\mathbf{x}, t)=\rho_{0}+\delta \psi(\mathbf{x}, t)$. By solving the linearized equation in Fourier space, the Fourier transform of $\delta \psi(\mathbf{x}, t)$, behaves as $\delta \hat{\psi}(\mathbf{k}, t) \sim \exp (\lambda(k) t)$, with:

$$
\lambda(k)=-D_{0} k^{2}\left(1+\frac{2 p J_{1}(k R)}{k R}\right),
$$

where $J_{1}$ is the first-order Bessel function. Instability, and therefore the formation of periodic patterns, appears for $\lambda>0$, which occurs for $p>p_{c} \approx 7.6$. This is the value obtained with the numerical simulations of the discrete model and the density equation. Note also that from the 
functional form of Eq. (7) it is clear that the mode of maximum growth scales as $1 / R$. We also note that the $D_{0}$ only fixes the relation between spatial and temporal scales, being the pattern transition, once fixed $R$, solely determined by $p$.

The continuum density equation helps us to understand the pattern formation in the original particle model. It arises as a deterministic instability of the homogeneous density.

\section{Non-local birth-death dynamics}

In the former section we presented a model where the non-locality acts on the random motion of the particles. No other kind of interaction among agents was considered and, therefore, the total number of particles was conserved. In this second part we consider a different particle model which is of the birth-death type. Now the non-locality comes to play on the particle reproduction rates, while their motility is constant and the same for all of them. Despite this rather different framework, analogous spatial structures, originated via the same mechanism (a deterministic instability of the density equation), appear here.

\subsection{Birth-death particle model}

The system consists initially in a set of $N_{0}$ particles randomly located in a two dimensional square of size $L \times L(L=1)$ and periodic boundary conditions. The dynamical evolution is the following: chose a particle at random (let us call it the particle $i$, at position $x_{i}$ ). Then, probabilities $\lambda_{i}$ and $\beta_{i}$, of reproduction and death respectively, are calculated as $\lambda_{i}=\max \left(\lambda_{0}-\right.$ $N_{R}(i) / N_{s}, 0$ ), and $\beta_{i}=\beta_{0} . \lambda_{0}$ and $\beta_{0}$ are positive constants (the same for all the particles), verifying that $\lambda_{0}+\beta_{0}=1, N_{s}$ is a saturation constant, and $N_{R}(i)$ is, as before, the number of other particles within a distance $R$ of the chosen one $i$. With the birth probability $\lambda_{i}$, a new particle is introduced in the system exactly at the location of the parent particle $i$, and with the death probability $\beta_{i}$, the particle $i$ disappears from the system. Repeat this process for all the particles in the system. Finally, each particle is moved with a small random displacement sampled from a Brownian motion with diffusion coefficient $D_{0}$.

This model was analyzed in [11] and two different transitions were identified:

- An absorbing phase transition with two phases in the statistically stationary regime: an empty phase, where all the particles have died, and an active phase, reached for large values of a control parameter, where there are many particles in the system. The bifurcation parameter is $\mu=\lambda_{0}-\beta_{0}$.

- A transition from homogeneous particle distribution to a periodic arrangement of particle clusters. This transition occurs in the active phase by decreasing the diffusion coefficient at fixed values of $\mu$ and $R$.

Detailed analysis of this model can be found in [11-14]. Here we focus in the analogies with the first particle model studied in Sect. 2 and therefore in the second of the transitions mentioned above. In Fig. 4 we show the distribution of particles at long times, when the system is in the active phase (large $\mu$ ) and two different values of the diffusion coefficient. For large $D_{0}$ the particles are randomly distributed, and for small $D_{0}$ they arrange in an hexagonal pattern of clusters. This behavior is analogous to the one found in the previous particle model, though here the dynamics is completely different: in addition to moving, particles are continuously appearing and disappearing from the system (birth-death dynamics), and their total number is not conserved. The mechanism of clustering and pattern formation here can also be understood from analysis of the mean-field density equation, and turns out to be also a deterministic linear instability of the homogeneous density.

\subsection{Continuum description}

Due to the varying number of particles the way to obtain a density equation for the model is not that obvious. A standard procedure (used in [11]) first divides the space in a number 

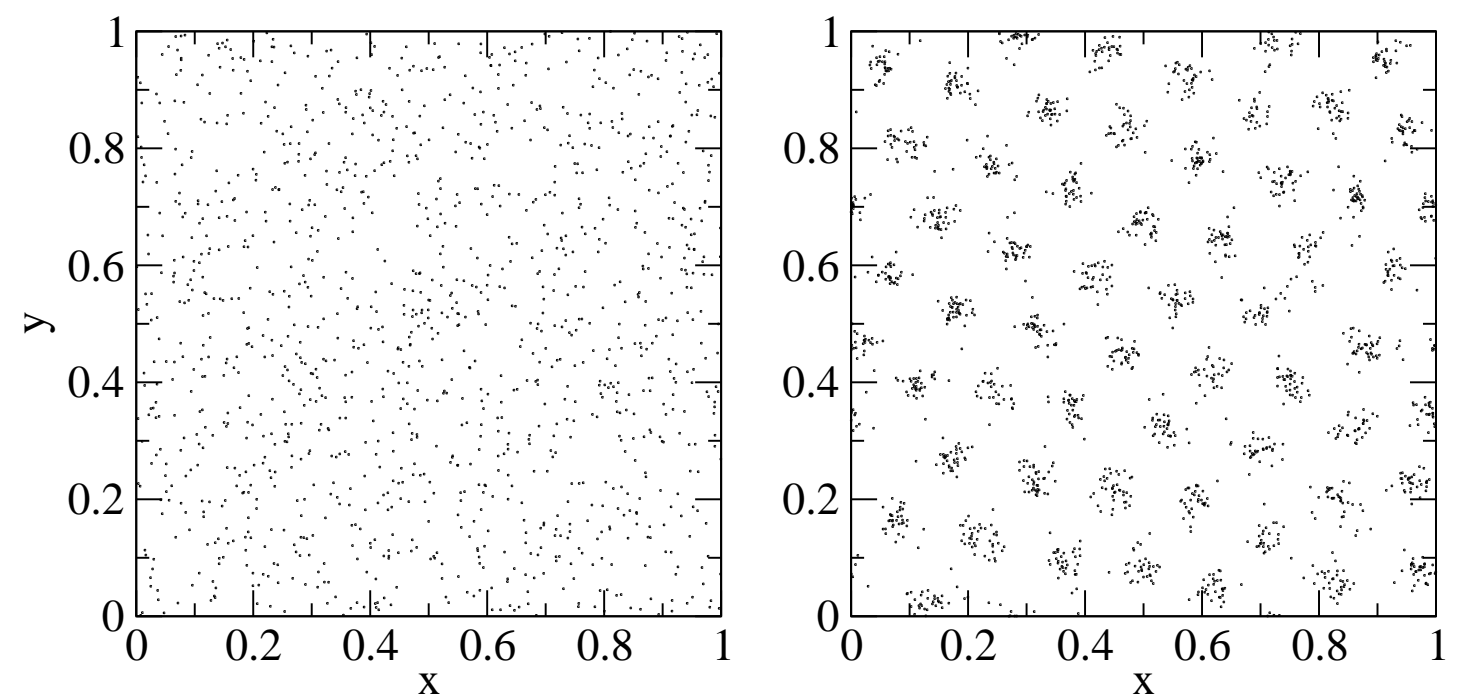

Fig. 4. Spatial distribution of particles under the birth-death dynamics at long times. Left is for $D_{0}=10^{-1}$, and right for $D_{0}=10^{-5}$. The rest of parameters are, in both plots, $\mu=0.8, R=0.1$, $N_{s}=50$, and $N_{0}=500$.

of cells, so that the state of the system is described by the number of particles inside each lattice cell. Then a Master Equation for the probability of having a number of particles in any of the cells can be written down. From this Master Equation one can, using for instance the mathematical formalism of annihilation-creation operators in a lattice, derive a Langevin equation for a continuous field whose expectation value, $\rho(\mathbf{x}, t)$, is the mean-field density of the system. The equation is:

$$
\partial_{t} \rho(\mathbf{x}, t)=D_{0} \nabla^{2} \rho(\mathbf{x}, t)+\mu \rho(\mathbf{x}, t)-\frac{1}{N_{s}} \rho(\mathbf{x}, t) \int_{|\mathbf{x}-\mathbf{r}|<R} d \mathbf{r} \rho(\mathbf{r}, t)
$$

This non-local density equation has also been obtained and analyzed in a different context [15]. At this mean-field level it is obvious that the critical value of $\mu$ for the absorbing phase transition (from death to active phase) is given by $\mu_{c}=0$. As usual in absorbing transitions, fluctuations shift the true critical point to larger $\mu$ values [12]. Numerical solutions however show that this equation retains correctly the pattern-formation dynamics of the discrete model. Fig. 5 shows the inhomogeneous density obtained from integration of (8) at small $D_{0}$. An hexagonal pattern very similar to the one in Fig. 3 (left) appears, with the same wavenumber as the corresponding particle model (Fig. 4, right).

Eq. (8) has a stationary homogeneous solution $\rho=\mu N_{s} /\left(\pi R^{2}\right)$. Linear stability analysis of this state can be performed, showing that perturbations of wavenumber $k$ grow as $\exp (\lambda(k) t)$, with

$$
\lambda(k)=-D_{0} k^{2}-\frac{2 \mu}{k R} J_{1}(k R) .
$$

The parameter values at which the maximum of the curve $\lambda(k)$ becomes positive, say $k_{M}$, gives the onset of pattern formation. The equations that identify them are:

$$
\begin{gathered}
\left.\frac{\partial \lambda}{\partial k}\right|_{k=k_{M}}=0, \\
\lambda\left(k_{M}\right)=0,
\end{gathered}
$$

which can be numerically solved and one obtains that $k_{M}=4.779 / R$, and that the critical value of $\mu$ for the pattern instability is given by

$$
\mu_{p}=185.192 \frac{D_{0}}{R^{2}} .
$$




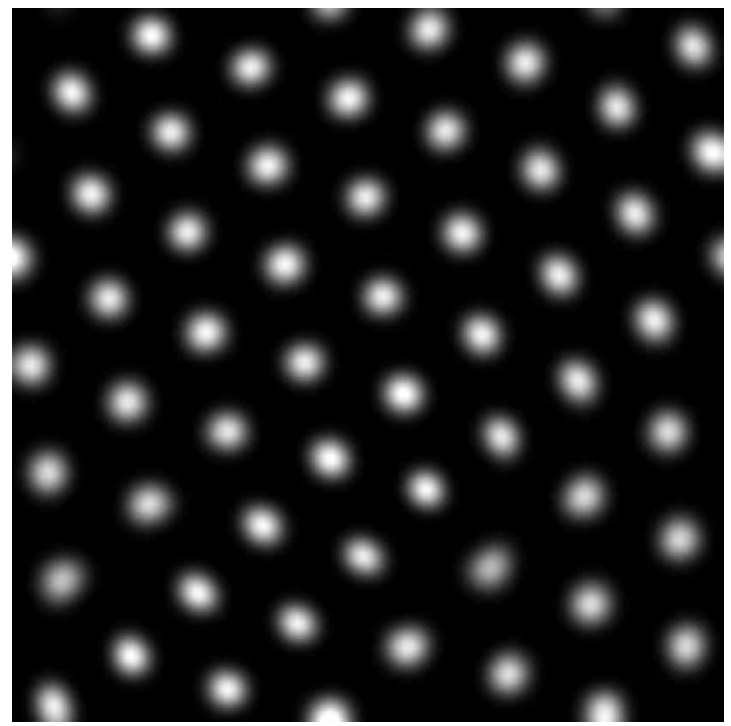

Fig. 5. Pattern obtained from the mean-field density equation (8) at moderately long times, when the density approaches the steady periodic state. Black is $\rho \approx 0$ and lighter grey levels are for high density values. Parameter values are $\mu=0.8, R=0.1, D_{0}=10^{-5}$, and $N_{s}=50$.

This confirms that for $\mu>\mu_{p}$ a spatial pattern emerges at wavenumber $k_{M}$. Note that at fixed $\mu>0$ one can cross this line of instability, and obtain a periodic structure, by decreasing $D_{0} / R^{2}$

Thus, important phenomenology of the particle model is qualitatively well described by the density equation. The value of the critical wavenumber is even quantitatively accurate [11]. However, as discussed in [12], the critical values of the parameters for the two transitions are not well reproduced in the mean-field approach. This contrast with the quantitative agreement obtained for the conserved-particle model discussed in Sect. 2. Fluctuations, coming from the discrete nature of the particles and neglected in the mean-field approximation, are thus more important in this second birth-death dynamic model.

\section{Discussion}

We have presented two rather different kinds of dynamics in which random walkers interact non-locally. Despite that interactions appear in the diffusivity for the first model and in the birth rates for the second, and that particle number remains constant in the first and fluctuates in the second, the same pattern forming phenomenon, leading to particle clusters arranged periodically with hexagonal symmetry, is observed in both cases. A density description allows the identification of the clustering phenomenon in both models as a deterministic pattern forming instability of the homogeneous density. In both cases the pattern periodicity is determined by the interaction range $R$. Despite these analogies, particle conservation in the first kind of dynamics seems to constrain fluctuations more strongly that under birth-death dynamics. As a consequence, the mean-field description gives more accurate approximations to the particle dynamics in the non-local density-dependent mobility model than in the case of birth and death. In fact there is at least one aspect of the particle dynamics in the non-conserved case which is completely absent at the mean field level: the existence of reproductive correlations [16]. They arise since, after each reproduction event, offspring is located at the same position as the parent. Because of this, it is observed that essentially all particles in a given cluster in Fig. 4 are descendants of a common ancestor. This phenomenon, and the fact that particles only travel a finite distance during their lifetime, gives rise to clustering even in the absence of interactions [16]. In the present interacting model, it forces the cluster width to be limited by the particle 
diffusivity [14]. A striking consequence of this arises when the interaction range $R$ is the full system size: Particles remain concentrated in a single cluster of size determined by diffusion and death rate [14], whereas the mean-field equation predicts an homogeneous solution.

Non-locality appears quite naturally in interacting particle models. These systems provide fascinating manifestations of nonlinear phenomena such as pattern formation, which are more traditionally considered in the context of continuous models. As shown here, however, the methodologies developed in the continuous approaches provide very useful tools to address the particle phenomenology.

We were indebted to Prof. Carlos Pérez-García for his many insights in the concepts and techniques of pattern formation phenomena. Financial support from MEC (Spain) and FEDER through project CONOCE2 (FIS2004-00953) is greatly acknowledged. C.L. is a Ramón y Cajal fellow of the Spanish MEC.

\section{References}

1. G. Flierl, D. Grunbaum, S. Levin, and D. Olson, J. Theor. Biol. 196, (1999) 397; A. Mogiler and L. Edelstein-Keshet, J. Math. Biol. 38, (1999) 534 ; F. Cecconi, M. Marsili, J. R. Banavar, and A. Maritan, Phys. Rev. Lett. 89, (2002) 088102; C. Topaz and A. Bertozzi, SIAM J. Appl. Math. 65, (2004) 152; Y. E. Maruvka and N. M. Shnerb, Phys. Rev. E 73, (2006) 011903

2. T. Vicsek, A. Czirók, E. Ben-Jacob, I. Cohen, and O. Shochet, Phys. Rev. Lett. 75, (1995) 1226

3. D. D. Holm and V. Putkaradze, Phys. Rev. Lett. 95, (2005) 226106 ; D. D. Holm and V. Putkaradze, nlin.PS/0506020

4. C. López, Phys. Rev. E 72, (2005) 061109

5. C. López, Phys. Rev. E 74, (2006) 012102

6. J.D. Murray, Mathematical Biology I, (Springer, Berlin 2005)

7. N. G. van Kampen, Stochastic Processes in Physics and Chemistry, (Elsevier North-Holland, Amsterdam 1981)

8. W. Horsthemke and R. Lefever, Noise-induced transitions, (Springer, Berlin 1984)

9. D. S. Dean, J. Phys. A 29, (1996) L613

10. U. Marini Bettolo Marconi and P. Tarazona, J. Chem. Phys 110, (1999) 8032

11. E. Hernández-García and C. López, Phys. Rev. E 70, (2004) 016216

12. C. López and E. Hernández-García, Physica D 199, (2004) 223-234

13. E. Hernández-García and C. López, Physica A 356, (2005) 95-99

14. E. Hernández-García and C. López, J. Phys.: Condens. Matter 17, (2005) S4263-S4274

15. M. A. Fuentes, M. N. Kuperman, and V. M. Kenkre, Phys. Rev. Lett. 91, (2003) 158104 ; 70, (2004) 016216 ; M. G. Clerc, D. Escaff, and V. M. Kenkre, Phys. Rev. E 72, (2005) 056217

16. W.R. Young, A.J. Roberts and G. Stuhne, Nature 412, (2001) 328 